\title{
Análise Espacial: Conceito, Método e Aplicabilidade
}

\author{
Adriana Giavoni ${ }^{1}$ \\ Universidade Católica de Brasilia \\ Álvaro Tamayo \\ Universidade de Brasilia
}

\begin{abstract}
Resumo
A proposta deste artigo foi introduzir um novo tipo de análise - a análise espacial. Baseada em conceitos matemáticos inseridos na análise fatorial, esta análise permite avaliar espacialmente o objeto de estudo. Utilizando os conceitos de dimensão e base vetorial, pôde-se representar e avaliar espacialmente o esquema masculino que compõem o Inventário dos Esquemas de Gênero do Autoconceito (IEGA). Por ser uma estrutura tridimensional, este esquema foi representado graficamente. Análises algébricas podem ser realizadas para estruturas espaciais superiores a três dimensões. A análise espacial permite integrar as dimensões que constituem o objeto de estudo, formando uma estrutura unitária; avaliar o objeto de estudo a partir de novas variáveis - as variáveis espaciais e avaliar uma estrutura hierárquica. Para uma avaliação mais completa do objeto, a análise espacial deve ser realizada conjuntamente com as análises parcial e total.

Palavras-chave: Esquemas de gênero; estrutura espacial.
\end{abstract}

Spatials analysis: Concept, Method and Applicability

\begin{abstract}
The purpose of this article was to introduce a new analysis - the spatial analysis. Based on mathematical concepts inserted in factor analysis, this kind of analysis spatially represents and evaluates a study subject. Using the dimension and vector base concepts, the masculine scheme that composes the SelfConcept Gender Schemas Inventory (IEGA) was spatially represented and evaluated. Because this scheme is tridimensional, it could be graphically represented. Above three dimensions, only the algebric analysis could be done. The spatial analysis allows to integrate all dimensions that perform a study subject in a whole; evaluate a study subject from the new perspective - the spatial variables and evaluate a hierarchical structure. For the full evaluation of the subject, the spatial analysis should be carried out with another analysis, such as total and partial analysis.

Keywords: Gender squemas; spatial structure.
\end{abstract}

A análise de um objeto de estudo qualquer pode ser efetuada a partir de várias perspectivas, que diferem quanto à dimensionalidade, caminhando da unidimensionalidade a multidimensionalidade. Ao nível psicométrico poder-se-ia definir uma análise unidimensional como aquela em que um único escore representa o objeto de estudo, enquanto em uma análise multidimensional, o objeto de estudo encontrase representado por vários escores, escores estes que definem os diferentes aspectos que o compõem.

Apesar de permitir uma melhor avaliação e compreensão do objeto de estudo, as análises multidimensionais tendem a fragmentá-lo em seus distintos aspectos, perdendo-se a composição global ou unitária do mesmo. Torna-se, portanto, necessário desenvolver um novo tipo de análise que permita uma avaliação integral, na qual as dimensões tornar-se-ão partes constitutivas do corpo do objeto de

\footnotetext{
${ }^{1}$ Endereço para correspondência: SHIS QL18 Conjunto 06 Casa 15, Lago Sul, 71650 065, Brasília, DF. Fone (61) 366-4203.E-mail: adriana@pos.ucb.br
}

estudo, podendo este vir a ser representado e avaliado espacialmente, como uma unidade. Esta será a proposta deste trabalho. Desenvolver conceitos matemáticos que, ao serem aplicados à análise fatorial, permita representar e avaliar o objeto de estudo a partir de uma nova ótica - a análise espacial.

A análise fatorial está fundamentada sobre conceitos da álgebra linear. Estes conceitos são fundamentais para a compreensão do que é um fator e de quando este pode ser considerado como uma dimensão de um objeto de estudo.

O primeiro conceito que será aqui tratado é o conceito de dimensão (Boldrini, Costa, Ribeiro \& Wetzler, 1980). Em álgebra linear, o conceito de dimensão está vinculado a um vetor que satisfaz duas propriedades básicas: a) Gerar outros vetores e b) Ser linearmente independente. Um vetor gera outros vetores, quando ele pode ser considerado como uma unidade básica constituinte dos demais vetores. Como exemplo, poder-se-ia citar o vetor i de tamanho 1 (Figura 1). Este vetor poderá gerar infinitos vetores que serão combinações lineares dele mesmo, ou seja, ele multiplicado por ele mesmo $n$ vezes $(v=n$. i ). Assim, a partir dele pode- 
se obter vários vetores que tenham o dobro, triplo, ... de seu tamanho. A Figura 1 apresenta os vetores i e j e a exemplificação de alguns vetores por eles gerados.

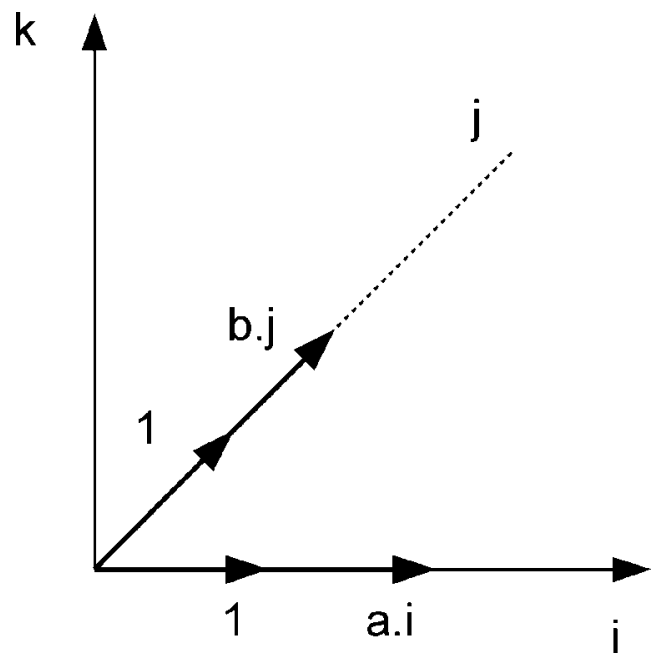

Figura 1. Vetores i e j e demais vetores por eles gerados.

Um vetor é considerado linearmente independente quando ele, como unidade básica, não apresentar relação linear com outros vetores que também são unidades básicas de um espaço vetorial. Para que um vetor seja considerado linearmente independente em relação a outro vetor, qualquer tentativa de combinação linear entre eles deve gerar um vetor nulo. Considerando o par ordenado do vetor i como $(1,0)$ e do vetor $j$ como $(1,1)$, obtém-se a seguinte expressão matemática ao igualá-los ao vetor nulo $(0,0):[a \cdot(1,0)+b \cdot(1,1)=(0,0)]$. Desenvolvendo a equação temos: $(a, 0)+(b, b)=(0,0)$, o que resulta no seguinte sistema de equações: $\mathrm{a}+\mathrm{b}=0$ e $0+\mathrm{b}=0, \operatorname{logo}, \mathrm{b}=0$. Se $b=0$, então, substituindo b na equação 1 , temos que $\mathrm{a}=0$. Assim, qualquer combinação linear entre os vetores i e j resultará no valor nulo $(0,0)$. Pode-se afirmar, portanto, que os vetores i e j são linearmente independentes.

Assim, se um vetor qualquer for uma unidade básica geradora de outros vetores e, este mesmo vetor, for linearmente independente em relação aos demais vetores, que também são unidades básicas, então, este vetor formará uma dimensão. Na Figura 1, os vetores i e j podem ser considerados como dimensões de um espaço vetorial.

Quando, em um espaço vetorial, só se encontrarem vetores considerados como unidades básicas e linearmente independentes entre si, então, teremos um segundo conceito importante denominado de base vetorial. Uma base vetorial é formada por um conjunto finito de vetores, que são unidades básicas e independentes entre si. Cada vetor formará uma dimensão e o conjunto de vetores gerará o espaço vetorial. Assim, por exemplo, a base vetorial que gera um espaço tridimensional é formada por três vetores que são unidades básicas e linearmente independentes entre si.

Este conceito de base vetorial está intimamente relacionado à técnica da análise fatorial. Segundo Harman (1976), o conjunto de fatores extraídos em uma análise fatorial representa uma base vetorial, ou seja, cada fator formará uma dimensão da base vetorial. Recordando que uma base vetorial é formada por vetores que satisfazem duas propriedades básicas: 1) gerar outros vetores, e 2) ser linearmente independente, analisar-se-á a aplicação destas duas propriedades à análise fatorial.

1) Gerar outros vetores: $\mathrm{Na}$ análise fatorial, cada fator representa um conjunto de variáveis que estão correlacionadas entre si. Traduzindo para a linguagem vetorial, temos: a) as variáveis são vetores em um espaço vetorial; $b$ ) variáveis com altas correlações entre si permanecem próximas no espaço vetorial, formando um conjunto; c) cada conjunto é representado por um único vetor. Este vetor é a unidade básica constituinte dos demais vetores, ou seja, este vetor é capaz de gerar todos os demais vetores que formam o conjunto e d) os vetores considerados como unidades básicas geradores de conjuntos de variáveis são denominados de fatores. A Figura 2 apresenta os vetores i, j e k que geram três conjuntos de variáveis. Estes três vetores são os fatores extraídos na análise fatorial.

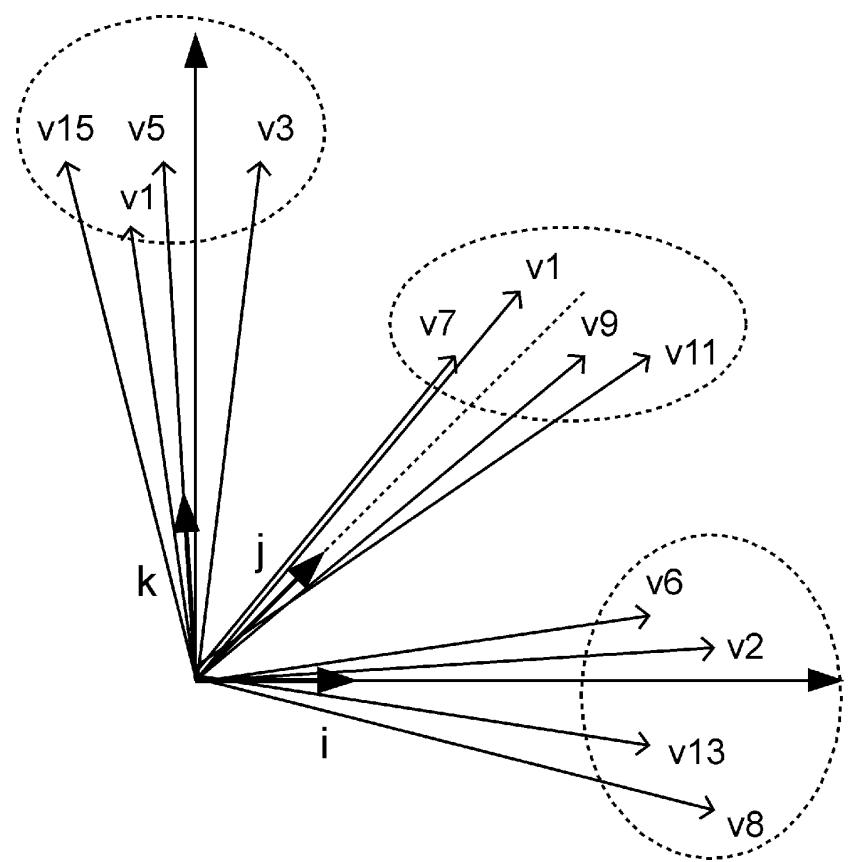

Figura 2. Vetores i, j e k gerando três conjuntos de variáveis.

Assim, se em uma análise fatorial forem extraídos três fatores, teremos, em um espaço vetorial, três vetores. Estes vetores serão unidades básicas representantes de três 
conjuntos de variáveis. Este três vetores são capazes de gerar todas as variáveis deste espaço vetorial. Desta forma, a primeira propriedade que define o conceito de dimensão encontra-se devidamente aplicada à análise fatorial.

2) Ser linearmente independente: A técnica da análise fatorial nos permite saber, através da Matriz de Correlação entre os Fatores, se os fatores são independentes entre si. Segundo Tabachnick e Fidell (1996) pode-se considerar os fatores como independentes, quando estes apresentarem correlações iguais, ou inferiores, a 0,32. Traduzindo para a linguagem vetorial, isto nos assegura que os vetores, enquanto representantes dos fatores, não possuem relação linear entre si, ou seja, são linearmente independentes. Desta forma, a segunda propriedade que define o conceito de dimensão encontra-se devidamente aplicada à análise fatorial.

Há, entretanto, situações em que os fatores apresentamse correlacionados entre si, ou seja, são linearmente dependentes. Nestas situações, a segunda propriedade básica que define o conceito de dimensão não é satisfeita. Inicialmente, portanto, estes fatores não podem ser considerados como dimensões de um objeto de estudo. Vetorialmente, isto significa que ainda existe um vetor subjacente capaz de gerar estes vetores linearmente dependentes. Fatorialmente, estas unidades básicas subjacentes são denominadas de fatores de segunda ordem, terceira ordem e, assim, sucessivamente.

Chega-se, portanto, à definição de base vetorial, pois se os fatores, enquanto vetores, são unidades básicas geradoras de conjuntos de variáveis, e se, além disso, são linearmente independentes entre si, então, temos uma base vetorial. Sendo uma base vetorial, cada um destes fatores formará uma dimensão do espaço vetorial.

Uma base vetorial com três fatores gera um espaço tridimensional, assim como, uma base vetorial de $\mathrm{n}$ fatores gera um espaço n-dimensional. Espaços bidimensionais geram formas espaciais planas, enquanto espaços ndimensionais geram formas espaciais definidas por volumes. As formas geradas no espaço vetorial são as representações espaciais do objeto de estudo.

A título de ilustração, aplicar-se-á os conceitos de dimensão e base vetorial no Inventário dos Esquemas de Gênero do Autoconceito (IEGA) (Giavoni \& Tamayo, 2000). Este instrumento avalia os esquemas masculino e feminino do autoconceito. Constituído por 83 itens, subdivididos em duas escalas - escala masculina (41 itens) e escala feminina (42 itens), o IEGA permite avaliar como os construtos sociais de masculinidade e feminilidade encontram-se estruturados na subjetividade individual. Os fatores das escalas masculina e feminina representam os diferentes aspectos que formam os esquemas masculino e feminino, presentes no autoconceito.
A escala masculina é composta por quatro fatores, denominados de: Negligência, Racionalidade, Ousadia e Agressividade. Aplicando os conceitos matemáticos vistos anteriormente pode-se afirmar que estes quatro fatores representam quatro conjuntos de variáveis. Por exemplo, o fator Racionalidade agrupa as variáveis: racional, realista, objetivo(a), competitivo(a), etc. Como cada fator representa um conjunto de variáveis, pode-se afirmar que estes satisfazem a primeira propriedade que define o conceito de dimensão, ou seja, gerar outros vetores.

Analisando a matriz de correlações entre os fatores observa-se que os fatores Negligência e Agressividade encontram-se correlacionados $(r=-0,38)$, resultando em um fator de segunda ordem. Vetorialmente, isto significa dizer que estes dois vetores são linearmente dependentes, sendo gerados por um vetor subjacente. Este vetor subjacente é o fator de segunda ordem, denominado de Indiferença.

Assim, os três fatores que satisfazem as duas propriedades básicas que definem o conceito de dimensão são os fatores: Racionalidade, Ousadia e Indiferença. Por serem dimensões, estes três fatores formam uma base vetorial tridimensional cuja forma será a representação espacial do esquema masculino. A Figura 3 apresenta, graficamente, a base vetorial que forma o esquema masculino.

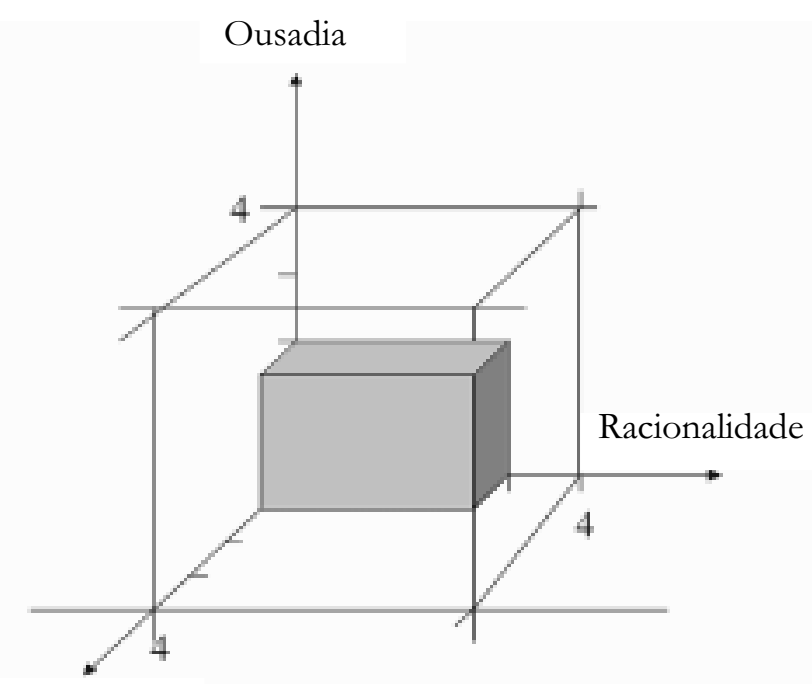
Indiferença

Figura 3. Representação espacial do esquema masculino.

Várias formas geométricas podem ser geradas em um espaço tridimensional, mas como a escala utilizada no IEGA era de cinco pontos, variando de 0 a 4 , pode-se definir que, em módulo, os vetores que formam a base vetorial variarão de 0 a 4 . Assim, ao assumirem as suas magnitudes máximas, a forma gerada no espaço vetorial será a de um cubo. $\mathrm{O}$ 
cubo, portanto, será a forma espacial do esquema masculino quando este atingir o seu nível máximo de desenvolvimento.

Vamos supor que um sujeito hipotético obtenha o escore 2 para o fator Ousadia, o escore 1 para o fator Indiferença e o escore 3 para o fator Racionalidade. Estes escores representam as magnitudes que os vetores assumirão no espaço vetorial. A Figura 3 apresenta internamente a representação espacial do esquema masculino deste sujeito hipotético. Observe que, para este sujeito, o esquema masculino assume a forma de um paralelepípedo. Nota-se, portanto, que para cada sujeito, o esquema masculino assumirá uma formatação diferente no espaço vetorial, variando quanto à forma, volume, nível de desenvolvimento, etc.

Observe que os fatores que formam a base vetorial representam as diferentes dimensões que compõem o corpo do objeto de estudo, no caso, o esquema masculino e, além disso, a forma gerada pela base vetorial será a própria representação espacial do corpo do objeto de estudo. Através da base vetorial, portanto, pode-se conhecer quais são as dimensões que compõem o corpo do objeto de estudo, assim como, é possível avaliá-lo espacialmente. O conhecimento da base vetorial permite, portanto, efetuar três tipos de análises, denominadas de: análise parcial, análise total e análise espacial. A análise parcial avalia cada dimensão do objeto de estudo. A análise total avalia o conjunto de dimensões que o compõem, resultando em um perfil do mesmo e a análise espacial avalia a estrutura espacial do objeto, gerada por suas distintas dimensões. Observe que a análise espacial é um tipo de análise decorrente da aplicação dos conceitos de álgebra linear que estão inseridos na análise fatorial. A análise espacial permite abordar o objeto de estudo a partir de novos ângulos, abrindo a perspectiva de se avaliar novas variáveis resultantes da combinação de suas dimensões. Nesta análise, aspectos (ou dimensões) antes desconexos e tratados independentemente, reúnem-se gerando uma forma única - a forma espacial. As novas variáveis - variáveis espaciais, resultantes deste tipo de análise, levam em consideração a constituição de toda estrutura. Como analogia poder-se-ia citar o corpo humano. Este é constituído por diversos sistemas (circulatório, digestivo, pulmonar, etc.) que podem ser analisados individualmente (análise parcial) ou em conjunto (análise total), mas a análise em conjunto não reflete o homem enquanto forma e, tampouco, enquanto variáveis resultantes da combinação e interação de seus distintos aspectos. Variáveis corporais como peso, estatura, diâmetros, perímetro e circunferências corporais são variáveis avaliadas a partir da estrutura espacial humana e não correspondem, apenas, ao somatório de distintas partes do corpo. Desta forma, a análise espacial permite avaliar variáveis espaciais (áreas planas, volumes, níveis de desenvolvimento, formas, etc.) que diferem, simplesmente, do perfil do objeto quando este é analisado como um conjunto de dimensões independentes.

Com relação aos objetos de estudo que comportam bases vetoriais superiores a três dimensões, estes não podem ser representados graficamente, mas isto não inviabiliza as análises espaciais. Por serem resultantes de cálculos algébricos, estas análises podem ser efetuadas sobre bases vetoriais de $\mathrm{n}$ dimensões.

Por serem decorrentes da composição multidimensional do objeto de estudo, as variáveis espaciais podem ser utilizadas como dimensões de uma nova base vetorial, permitindo, como conseqüência, avaliar uma estrutura hierárquica, como o autoconceito. Concebido como uma estrutura hierárquica e multidimensional, o autoconceito é representado no ápice de sua estrutura hierárquica pelo autoconceito geral, o qual se ramifica em distintos aspectos: self físico, self social, self familiar, self acadêmico, etc. Estes, por sua vez, ramificam-se novamente em subestruturas. $\mathrm{O}$ self físico, por exemplo, decompõe-se em dois novos aspectos: performance física e aparência física. A Figura 4 apresenta uma representação desta estrutura hierárquica e multidimensional que é o autoconceito.

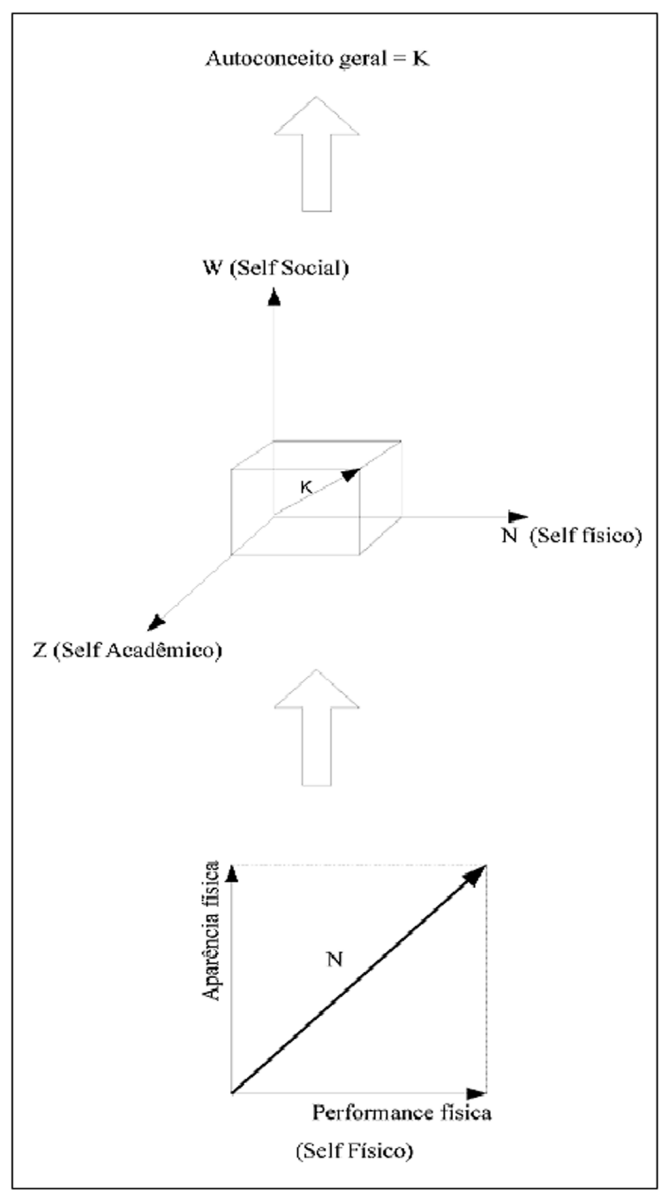

Figura 4. Representação parcial da estrutura hierárquica do autoconceito. 
Considerando os aspectos performance física $(\mathrm{PF})$ e aparência física (AF) como dimensões do self físico, estas duas dimensões gerarão uma base vetorial bidimensional, cujo vetor resultante poderá ser utilizado como vetor representativo do self físico em uma nova base vetorial. Este vetor resultante avalia o comprimento da estrutura espacial e pode ser obtido através do teorema de Pitágoras $\left(\mathrm{N}^{2}=\mathrm{AF}^{2}+\mathrm{PF}^{2}\right)$. Procedimento matemático semelhante pode ser aplicado a estruturas espaciais iguais ou superiores a três dimensões, sendo que a diferença está no acréscimo do número de dimensões. Assim, para calcular o comprimento de um vetor que parte da origem até um ponto específico de um espaço tridimensional, adiciona-se uma variável ao teorema de Pitágoras $\left(\mathrm{d}^{2}=\mathrm{X}^{2}+\mathrm{Y}^{2}+\mathrm{W}^{2}\right)$.

A nova base vetorial, de nível superior, será composta pelo vetor representativo do selffísico e pelos demais vetores representativos, oriundos de bases vetoriais específicas que formam os selves - social, familiar, acadêmico, etc...

Por serem variáveis resultantes das dimensões que compõem a base vetorial, as variáveis espaciais originam-se de expressões matemáticas específicas e não, simplesmente, do mero somatório como muitas vezes presencia-se nas escalas psicométricas e estudos psicológicos. O critério para a seleção das variáveis espaciais a serem avaliadas dependerá do objeto de estudo que está sendo analisado e da relevância do estudo da variável espacial no contexto teórico abordado.

Diante de um objeto de estudo qualquer, pode-se, portanto, avaliá-lo parcialmente, obtendo-se escores representativos de cada dimensão (análise parcial). Por exemplo, pode-se obter a média do fator performance física. A seguir, pode-se representar graficamente o conjunto de escores obtidos em todas as dimensões (análise total), retratando o perfil do sujeito ou grupo avaliado e, finalmente, pode-se avaliar espacialmente a estrutura (análise espacial), obtendo-se escores que levam em consideração todas as dimensões da estrutura espacial e que poderão ser utilizados como vetores constituintes de novas estruturas espaciais.

Resta ainda uma última colocação quanto às bases vetoriais. Como se está utilizando o estudo de Giavoni e
Tamayo (2000) a título de exemplificação, considerou-se que os fatores que compõem a base vetorial do esquema masculino fossem ortogonais entre si, ou seja, encontramse dispostos a 90 graus no espaço vetorial. Isto porque, a segunda propriedade que define uma base vetorial - ser linearmente independente, não implica necessariamente que os fatores sejam ortogonais. Como foram extraídos através de rotações oblíquas, estes fatores não estão dispostos ortogonalmente no espaço vetorial. São as rotações do tipo varimax que asseguram este tipo de disposição espacial.

Em álgebra linear há um teorema que demonstra que quando os vetores estão dispostos ortogonalmente no espaço vetorial, pode-se afirmar que estes vetores são linearmente independentes entre si. Entretanto, não é o ângulo de 90 graus que define a independência entre os vetores. Pode-se encontrar dois vetores linearmente independentes e que não sejam ortogonais entre si, como, por exemplo, os vetores i e j da Figura 1 que estão dispostos a 45 graus e são, como demonstrado anteriormente, linearmente independentes. Para que os fatores (ou dimensões) que formam a base vetorial do esquema masculino venham a assumir uma disposição ortogonal, necessitar-se-ia realizar uma nova extração fatorial do tipo varimax, na qual seriam incluídas apenas as variáveis que compõem as dimensões desta base vetorial.

\section{Referências}

Boldrini, J. L., Costa, S. I. R., Ribeiro, V. L. F. F. \& Wetzler, H. G. (1980). Álgebra Linear. São Paulo: Harper \& Row do Brasil.

Giavoni, A. \& Tamayo, A (2000). Inventário dos esquemas de gênero do autoconceito. Psicologia: Teoria e Pesquisa, 16, 175-184.

Harman, H. H. (1976). Modern factor analysis. Chicago: University of Chicago Press.

Tabachnick, B. G. \& Fidell, L. S. (1996). Using multivariate statistics. California State University: HaperCollins College.

Sobre os autores

Adriana Giavoni é Doutora em Psicologia pela Universidade de Brasília (UnB) e Professora do Programa de Pós-graduação em Educação Física da Universidade Católica de Brasília (UCB). Álvaro Tamayo é Doutor pela Universidade de Louvain e Professor no Instituto de Psicologia da Universidade de Brasília (UnB). É consultor do CNPq. 